Faculty of Mathematical Sciences

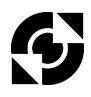

University of Twente

The Netherlands
P.O. Box 217

7500 AE Enschede

The Netherlands

Phone: +31-53-4893400

Fax: +31-53-4893114

Email: memo@math.utwente.nl

www.math.utwente.nl/publications

Memorandum No. 1608

Analysis of a feedback fluid model

for heterogeneous TCP sources

N. van Foreest, M. Mandjes

AND W.R.W. SCHEINHARDT

DeCEMBer 2001

ISSN 0169-2690 


\title{
Analysis of a Feedback Fluid Model for Heterogeneous TCP Sources
}

\author{
Nicky van Foreest ${ }^{\star}$, Michel Mandjes ${ }^{\ddagger \star}$, Werner Scheinhardt ${ }^{\star \ddagger}$ \\ * Faculty of Mathematical Sciences \\ University of Twente \\ P.O. Box, 7500 AE, Enschede, The Netherlands \\ \{n.d.vanforeest,m.r.h.mandjes, w.r.w.scheinhardt\}@math.utwente.nl \\ $\ddagger$ Center for Mathematics and Computer Science (CWI) \\ P.O. Box 94079, 1090 GB, Amsterdam, The Netherlands \\ \{michel.mandjes,w.r.w.scheinhardt\}@cwi.nl
}

February 15, 2002

\begin{abstract}
In this paper we consider a bottleneck link and buffer used by one or two fluid sources that are subject to feedback. The feedback is such that the model describes the behavior of the Transport Control Protocol as used in the Internet. During overflow, the buffer sends negative feedback signals to the sources to indicate that the sending rate should be reduced. Otherwise the buffer sends positive signals so as to increase the rate. In this context we find closed form expressions for the solution of the one-source case. The two-source case extends the single-source model considerably: we can control the behavior and parameters of each source individually. This enables us to study the impact of these parameters on the sharing of links and buffers in the Internet. For the two-source case we solve the related two-point boundary value problem in the stationary case. We also establish a numerically efficient procedure to compute the coefficients of the solution of the differential equations. The numerical results of this model are presented in an accompanying paper.
\end{abstract}

2000 Mathematics subject classification: 60K25 (primary), 68M20 (secondary)

Keywords and Phrases: fluid models, feedback, heterogeneous sources, TCP, shared fluid buffer

\section{Introduction}

The Internet consists of a set of resources, i.e., links and buffers, to transfer data packets from a source to a destination. To use these resources efficiently the source and destination, i.e., the end systems, run a congestion control protocol: TCP (Transport Control Protocol). TCP regulates the traffic rate of the source roughly as follows. A source injects packets into the network, which delivers these packets at the destination. The TCP receiver replies for each correctly received packet by sending an acknowledgment back to the source. As long as all sent packets arrive, the source can increase its rate linearly in time. At some point in time the packet rate (more precisely, the aggregate rate of many sources) exceeds the capacity of a bottleneck link. The buffer in front of the link starts to fill and will eventually overflow. This results in packet loss. Once the destination discovers this loss, it signals the sender. The sender then reduces its rate exponentially fast in time, usually by a factor two per roundtrip time (The roundtrip time is the time between sending a packet and receiving its acknowledgment). The sender continues to decrease its output rate until the congestion is removed. This dynamic scheme of the source rate is known as linear increase/multiplicative decrease.

Clearly, one of the key challenges in the Internet is to optimize the control algorithms of TCP and the network equipment such that the throughput along source-destination paths is maximized. Moreover, the 
scarce capacity should be divided among the users in some fair way, see $[8,9,14]$ for interesting discussions of fairness. Traditionally, simulation, and experiments have been the tools for examining these aspects of TCP. There is, however, a need for mathematical models to understand and (approximately) quantify the relative impact of the network and source parameters on link sharing and utilization. Recently, several efforts have been directed at modeling TCP to analyze its performance, e.g., [3, 15, 4].

Generally speaking, these models have two important shortcomings. First and foremost they usually study the behavior of the sources in response to the dynamically varying network conditions (in particular the way loss occurs). However, they do not take into account the opposite relation, i.e., the fact that network conditions are a result of the behavior of the sources. It may be clear that this can have a considerable impact on the validity of the results obtained. The second drawback of existing models is that they only allow to vary a small subset of the network and source parameters, while the other parameters are assumed to be constant.

This paper develops and analyzes a model that does not suffer from these shortcomings. We approximate the output stream of a TCP source as fluid so that the bottleneck buffer behaves as a fluid queue. In a companion paper [5] we provide detailed motivation and background for the accuracy of this approximation. There we also show that the model enables to examine the performance and fairness for the case one or two TCP sources connect to a bottleneck link via a FIFO buffer. In particular we study the impact and interaction of various system parameters such as source rates, buffer size, etcetera.

Literature. Fluid models have enjoyed quite some popularity in the literature; in particular Markov modulated fluid queues have been applied in the field of telecommunication, see e.g. [10, 2, 20]; in particular we mention [18] and [11], which provide nice general introductions and references to the field. In these models the joint source behavior is described by a Markov process. While the sources need not be mutually independent, the joint source process should be independent of the buffer content. However, TCP sources react to congestion signals from the buffer (i.e., packet loss). Hence, there is a strong two-way dependency between the states of the buffer and the sources. Clearly, traditional fluid models cannot be used to capture this intricate type of interaction.

The resolution is to be found in so-called feedback fluid queues, which were first introduced in [16]; see also [1]. In a feedback fluid queue the buffer content behaves as a standard Markov-modulated fluid queue, but the state of the background (regulating) process is now governed by a continuous-time generator $Q(y)$ whose rates depend on the current buffer content $y$. In [13] and [17] the stationary distribution is found for a class of feedback fluid queues where this dependence is piecewise constant, i.e., where the background process has a fixed generator as long as the buffer content is between two thresholds, or remains at one of the thresholds.

Contribution. As a first step we model the interaction of a single TCP source with a buffer as a feedback fluid queue, fitting in the framework of [13] and [17]. The background process models the rate at which the source is allowed to send data. The typical TCP behavior is included as follows. When the buffer is not full, the sending rate increases according to a pure birth process ('linear increase'); when the buffer is full, the rate decreases according to another generator so as to reflect 'multiplicative decrease'. This specific structure allows to find the stationary distribution from which the performance measures can be computed efficiently. The model enables us to vary the buffer size, link rate, source rate, the roundtrip time, and the number of source states to investigate the influence of these parameters on throughput and system utilization.

The main concern of this paper is the case of two or more TCP sources. This is not a 'standard' feedback fluid model in the sense of the earlier mentioned references. In particular, we extend the description of the process with indicator variables that keep track of which source(s) have to decrease their sending rates in case the buffer is not full. This is needed because when congestion (i.e. a full buffer) is dissolved due to the adaptation of some of the sources, the other sources should still adapt their rates as well, after they have been notified by negative feedback signals that are underway. We analyze the consequences of this phenomenon for two sources. The generalization of the model and its analysis to the case with more than two sources is, at least conceptually, not difficult and therefore not treated explicitly in this article.

Our model compares favorably to simulation in that it takes very little time to compute the performance measures of the sources, and study the intrinsic properties of the flow control algorithms of TCP. A drawback of the analytical approach is that it depends on the computation of an eigenvector of a large matrix whose 
condition number, see [6], increases as a function of the number of source states and the size of the buffer. In actual practice the numerical analysis turns out to be quite robust. As a result we could obtain interesting insights about TCP's behavior for an acceptable range of parameters.

In this paper we focus on the formal analysis of the model: the derivation of the Kolmogorov differential equations together with boundary conditions, and the methods to find expressions for the stationary distribution and the performance measures of interest. The performance analysis itself is described in the companion paper, [5], where we also discuss the existing literature on TCP models and provide an interpretation of the model in the context of TCP. Specifically, we compute the impact of the parameters on the utilization, both of the system as a whole, and of each source separately so that we are also able to consider the issue of fairness.

Organization. In Section 2 we explain the model. Then, in Section 3, we analyze the single-source case. The description used is a bit different from the one in [13, 17], and in fact more illuminating in the sense that several equations can now easily be identified as probability flux equations. This insight is also helpful in Section 4 , where we treat the two-source case. The last part of Section 4 contains an outline of the performance evaluation of TCP based on the present study. This evaluation is fully presented in the companion paper [5]. Finally, Section 5 summarizes the results of the current paper.

\section{The Model}

For completeness' sake we summarize here the TCP source model as developed in [5]. First we describe the behavior of a single source. Then we extend this to multiple sources.

As mentioned previously, we model a TCP sender as a fluid source. The state of the source is described by a stochastic process $\{X(t)\} \equiv\{X(t), t \geq 0\}$ with state space $\mathcal{S}=\{1,2, \ldots, N\}$. X(t) controls the output rate: when $X(t)=i$, the source sends fluid at rate $i r$ into a buffer. This buffer has size $b$ and is served by a link with constant capacity $l$. The source parameter $r$ corresponds to the increase of the source rate while the buffer is not congested. To avoid trivialities, we suppose that $r<l<N r$; the first inequality ensures that the buffer is not continuously in a state of overload, the second that occasionally congestion will occur. In Figure 1 the model is shown graphically.

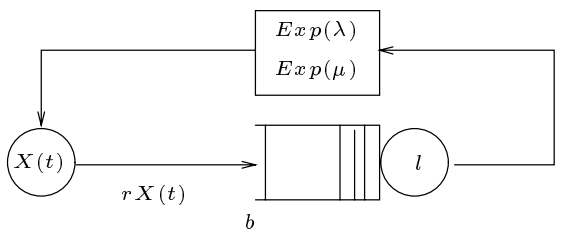

Figure 1: An overview of the model

The buffer sends positive and negative feedback signals to the source depending on the buffer content $\{C(t)\} \equiv\{C(t), t \geq 0\}$. As long as the buffer is not full, i.e., $C(t)<b$, the buffer sends positive signals to the source to indicate the successful transfer of data. When the source receives this feedback it increases its rate, i.e., $X(t)$ increases by one to $X(t)+1$ (except when $X(t)=N$, then it cannot increase any further) . This behavior of the source is known as linear increase. When the buffer becomes congested, i.e., $C(t)=b$, it sends negative signals to the source to notify that fluid is discarded. As a response, the source decreases its rate by half, that is, when $X(t)>1$ the state becomes $\lfloor X(t) / 2\rfloor$, the largest integer smaller than $X(t) / 2$ (when $X(t)=1$ it does not decrease further). We call this phase multiplicative decrease.

The time intervals between two consecutive positive or negative signals are assumed to be independent, identically distributed exponential random variables. The positive signals are, on the average, separated by $1 / \lambda$ time units. (The rate of the positive signals is therefore $\lambda$.) The negative signals are generated at rate $\mu$.

From the above it is clear that the source and buffer behavior are not independent. The source influences the buffer content by the rate at which it generates fluid; the buffer controls the source by sending positive and negative feedback signals. Hence we need a joint stochastic process $\{X(t), C(t)\} \in \mathcal{S} \times[0, b]$, i.e., the 
product of the source state and the buffer content, to characterize the system state. As the intervals between two signals of the same type are by assumption independent and exponentially distributed, the system state $\{X(t), C(t)\}$ evolves as a multivariate Markov process. This assumption simplifies the analysis considerably, to the extent that we can now associate Markov generators with the state transitions of the source. When $C(t)<b$, a generator $Q$ implements linear increase; when $C(t)=b$ the source should make multiplicative decrements according to a second generator $\widetilde{Q}$.

Figure 2 provides some further illustration of this behavior. Consider first the lower part of the figure. The source increases its transmission rate every time it receives a positive signal from the buffer. The interarrival time between these signals is exponentially distributed with parameter $\lambda$. In fact, its behavior is governed by the generator $Q$. At the very moment the buffer overflows - suppose this happens in state 4 - another generator $\widetilde{Q}$ becomes active. This change in generator is indicated by a switch from the lower to the upper part of the figure. There the source waits for an exponentially distributed time with parameter $\mu$, and then jumps to state 2. Since the buffer is still in overflow when the source enters this state, the source has to wait there for another negative feedback signal. When this is received, the source halves its state index for a second time, that is, jumps to state 1 . Now the net fluid rate into the buffer is negative. The buffer starts sending positive signals again, so the generator switches from $\widetilde{Q}$ to $Q$ again.

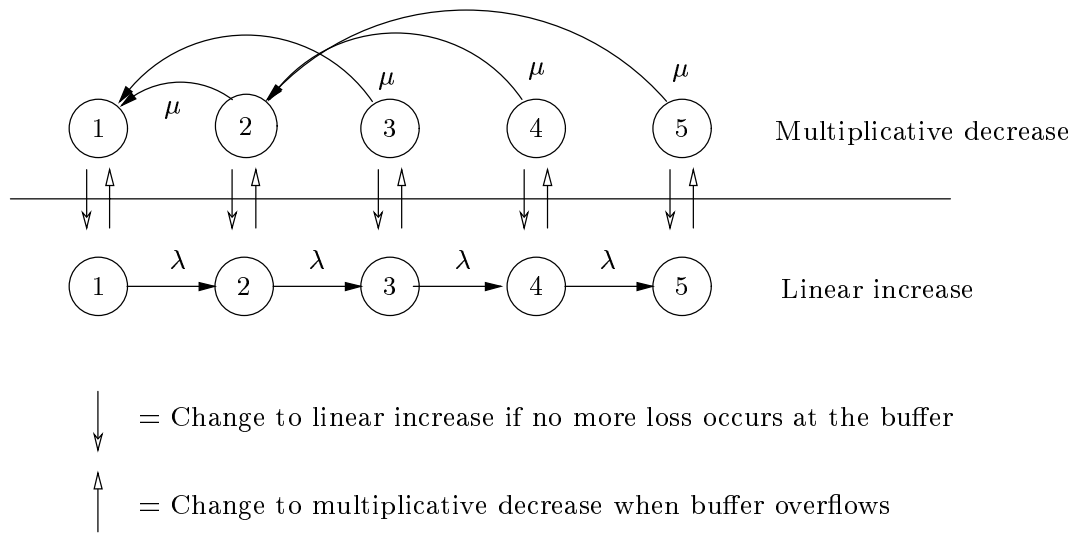

Figure 2: Behavior of a source with 5 states with $l=1.5$ and $r=1$.

Since we explicitly require that $r<l<N r$, the source rate alternates between periods in which its sending rate is higher and lower than the link rate. To distinguish between the underload and overload states, we define subsets of $\mathcal{S}: \mathcal{S}_{-}=\{i \in \mathcal{S} \mid i r<l\}$ and $\mathcal{S}_{+}=\{i \in \mathcal{S} \mid i r>l\}$. For technical reasons we assume that $l / r \notin \mathcal{S}$, that is, $l$ is not allowed to be an integer multiple of $r$. (See e.g. [19] how to handle systems for which $l / r \in \mathcal{S}$.) Consequently $\mathcal{S}=\mathcal{S}_{+} \cup \mathcal{S}_{-}$(and $\mathcal{S}_{-} \cap \mathcal{S}_{+}=\emptyset$ ). Furthermore we set $N:=|\mathcal{S}|$, $N_{-}=\left|\mathcal{S}_{-}\right|$and $N_{+}=N-N_{-}$.

In general we can have $J$ sources, labeled $j=1, \ldots, J$, that send fluid into one shared buffer. The behavior of each source individually is similar to the single-source case explained above. However, and this is crucial in the model, each source has its own set of parameters $r_{j}, \mu_{j}, \lambda_{j}, N_{j}$, which may differ from other sources. An interesting feature is now that sources may receive positive and negative signals at different rates and at different moments. A consequence is that after a period of congestion some sources still have to wait before they can increase their rate, while others have already started increasing their rate. (This phenomenon will be explained in more detail in Section 4.) To incorporate this situation, the state of a source needs to be expanded with an indicator variable $I_{i}(t)$. We set $I_{i}(t)=0\left(I_{i}(t)=1\right)$ when source $i$ receives positive (negative) signals from the buffer. The composite state space of these sources becomes therefore $\mathcal{S} \times \mathcal{I}$, where $\mathcal{S}=\mathcal{S}_{1} \times \cdots \times \mathcal{S}_{J}=\left\{\left(s_{1}, \ldots, s_{J}\right) \mid s_{j} \in \mathcal{S}_{j}\right\}$, and $\mathcal{I}=\mathcal{I}_{1} \times \cdots \times \mathcal{I}_{J}=\left\{\left(i_{1}, \ldots, i_{J}\right) \mid i_{j} \in\{0,1\}\right\}$. On $\mathcal{S} \times \mathcal{I}$ we define the process $\{\mathbf{X}(t), \mathbf{I}(t)\}=\left\{X_{1}(t), \ldots, X_{J}(t), I_{1}(t), \ldots, I_{J}(t)\right\}$. Now the system state space is written as $\{\mathbf{X}(t), \mathbf{I}(t), C(t)\}$. Note that while $\{\mathbf{X}(t), C(t)\}$ is not a Markov process, $\{\mathbf{X}(t), \mathbf{I}(t), C(t)\}$ is multivariate Markov. Analogous to the single-source requirement, we demand that $\sum_{j=1}^{J} r_{j}<l<\sum_{j=1}^{J} N_{j} r_{j}$. 


\section{Analysis of a Single Source}

We now analyze the case in which only one source uses the buffer. After fixing some notation in Section 3.1, we derive in Section 3.2 a set of coupled linear differential equations and generators that govern the dynamics of the source and buffer behavior. These equations reduce to a stationary system that should satisfy a number of boundary conditions. Finding these conditions will be the topic of Section 3.3. In Section 3.4 we explicitly calculate the solution. Finally Section 3.5 presents some results. The analysis borrows from the work in $[17,13]$. In the setting of the current paper explicit results can be derived. The extension to multiple sources, the topic of the next section, goes beyond $[17,13]$.

\subsection{Notation}

The source state $X(t)$ ascends as long as $C(t)<b$ (and $X(t)<N$ ), while it descends when $C(t)=b($ and $X(t)>1)$. To reflect this difference in behavior, we split the system state space $\mathcal{S} \times[0, b]$, into two mutually exclusive subsets. The first subset of the space corresponds to an uncongested buffer, $\mathcal{T}=\mathcal{S} \times[0, b)$. On $\mathcal{T}$ we define functions

$$
A_{i}(y, t)=\mathbb{P}\{X(t)=i, C(t) \leq y\}, \quad 0<y<b .
$$

The other subset of the state space is $\tilde{\mathcal{T}}=\mathcal{S} \times\{b\}$, with functions

$$
D_{i}(t)=\mathbb{P}\{X(t)=i, C(t)=b\} .
$$

Since the domain of $A(y, \cdot)$ is the open interval $(0, b)$, we define on the boundaries:

$$
\begin{aligned}
A_{i}(b, t) & =\lim _{y \uparrow b} A_{i}(y, t), & A_{i}(0, t) & =\lim _{y \downarrow 0} A_{i}(y, t), \\
\frac{\partial A_{i}(b, t)}{\partial y} & =\lim _{y \uparrow b} \frac{\partial A_{i}(y, t)}{\partial y}, & \frac{\partial A_{i}(0, t)}{\partial y} & =\lim _{y \downarrow 0} \frac{\partial A_{i}(y, t)}{\partial y} .
\end{aligned}
$$

It is clear that $\mathbb{P}\{X(t)=i\}=A_{i}(b, t)+D_{i}(t)$. To avoid possible confusion later in the paper, we set $A_{i}(y, t)=D_{i}=0$ if $i \notin \mathcal{S}$.

For later use we introduce the vector $\mathbf{A}(y, t)=\left(A_{1}(y, t), \ldots, A_{N}(y, t)\right)$, and, likewise, $\mathbf{D}(t)=\left(D_{1}(t), \ldots, D_{N}(y)\right)$. We need in our analysis the restrictions of these vectors to the regions $\mathcal{S}_{-}$and $\mathcal{S}_{+}$. Hence, let $\mathbf{A}_{-}=$ $\left(A_{1}, \ldots, A_{N_{-}}\right), \mathbf{A}_{+}=\left(A_{N_{-}+1}, \ldots, A_{N}\right)$, etc.

\subsection{The Differential Equations}

The system of differential equations that determines $\mathbf{A}$ is well-known, see e.g. [2, 18]; we summarize the derivation here. Introduce the shorthand $r_{i}=i r-l$, fix $y \in(0, b)$ and take $\Delta t$ sufficiently small. Then we find for $2 \leq i<N$

$$
A_{i}(y, t+\Delta t)=\lambda \Delta t A_{i-1}(y, t)+(1-\lambda \Delta t) A_{i}\left(y-r_{i} \Delta t, t\right)+o(\Delta t)
$$

The boundaries at $i=1$ or $i=N$ require some extra care. We set $A_{0}(y, t) \equiv 0$ when $i=1$; for the case $i=N$ the factor $1-\lambda \Delta t$ should be set to 1 . Now, expanding the second term on the right hand side in $\Delta t$, this becomes up to $o(\Delta t)$

$$
A_{i}(y, t+\Delta t)=\lambda \Delta t A_{i-1}(y, t)+(1-\lambda \Delta t)\left[A_{i}(y, t)-r_{i} \Delta t \frac{\partial A_{i}}{\partial y}(y, t)\right] .
$$

Collecting terms, dividing by $\Delta t$, and taking the limit $\Delta t \rightarrow 0$ we find in matrix form the Kolmogorov forward equations for the row vector $\mathbf{A}(y, t)$ :

$$
\frac{\partial \mathbf{A}(y, t)}{\partial t}=\mathbf{A}(y, t) Q-\frac{\partial \mathbf{A}(y, t)}{\partial y} R
$$


where

$$
Q=\left(\begin{array}{ccccc}
-\lambda & \lambda & & & 0 \\
& -\lambda & \lambda & & \\
& & \ddots & \ddots & \\
& & & -\lambda & \lambda \\
0 & & \cdots & & 0
\end{array}\right)
$$

and

$$
R=\left(\begin{array}{cccc}
r-l & & & 0 \\
& 2 r-l & & \\
& & \ddots & \\
0 & & & N r-l
\end{array}\right) .
$$

The generator $Q$ models the linear increase of the source's state as long as there is no loss at the buffer and $X(t)<N$.

For completeness sake we remark that we do not specify the behavior of $\mathbf{A}(y, t)$ on the boundary $y=0$. (The details are easy to provide.)

The derivation of the differential equations for $\mathbf{D}$ is more involved. Observe that, when $i \in \mathcal{S}_{+}$, there is a net probability flux into $\widetilde{\mathcal{T}}$ from 'below', i.e., from $\mathcal{T}$ due to overflows of the buffer, and from 'above', i.e, from higher states $D_{2 i}$ and $D_{2 i+1}$ into $D_{i}$, resulting from multiplicative decrements of the source state. From this it follows that for small $\Delta t$ and $i \in \mathcal{S}_{+}$

$$
\begin{aligned}
D_{i}(t+\Delta t)= & (1-\mu \Delta t) D_{i}(t)+\mu \Delta t\left[D_{2 i}(t)+D_{2 i+1}(t)\right] \\
& +(1-\lambda \Delta t) \mathbb{P}\left\{X(t)=i, b-r_{i} \Delta t<C(t)<b\right\}+o(\Delta t) \\
= & D_{i}(t)+\mu \Delta t\left[D_{2 i}(t)+D_{2 i+1}(t)-D_{i}(t)\right] \\
& +(1-\lambda \Delta t)\left[A_{i}(b, t)-A_{i}\left(b-r_{i} \Delta t, t\right)\right]+o(\Delta t) .
\end{aligned}
$$

$\left(D_{i} \equiv 0\right.$, if $i \notin \mathcal{S}$.) On $\mathcal{S}_{-}$there is a flux from $\widetilde{\mathcal{T}}$ to $\mathcal{T}$, and the term $A_{i}\left(b-r_{i} \Delta t, t\right)$ in the above equation should be replaced by $A_{i}\left(b+r_{i} \Delta t, t\right)$ since now $r_{i}<0$. We follow the same procedure as for $\mathbf{A}$, that is, collecting terms, etc., to obtain forward equations for $\mathbf{D}$ :

$$
\frac{\partial \mathbf{D}(t)}{\partial t}=\mathbf{D}(t) \widetilde{Q}+\frac{\partial \mathbf{A}(b, t)}{\partial y} R,
$$

where $\widetilde{Q}$ is a multiplicative decrement generator. The general structure of $\widetilde{Q}$ is written in terms of Kronecker's $\delta$, i.e., $\delta_{i j}=1$ if $i=j$ and 0 otherwise:

$$
\widetilde{Q}_{i j}=-\mu \delta_{i j}+\mu \delta_{i, 2 j}+\mu \delta_{i, 2 j+1}+\mu \delta_{i 1} \delta_{j 1},
$$

with $1 \leq i, j \leq N$. To make the general structure somewhat clearer, we include as an example for a source with $N=5$ :

$$
\widetilde{Q}=\left(\begin{array}{ccccc}
0 & 0 & 0 & 0 & 0 \\
\mu & -\mu & 0 & 0 & 0 \\
\mu & 0 & -\mu & 0 & 0 \\
0 & \mu & 0 & -\mu & 0 \\
0 & \mu & 0 & 0 & -\mu
\end{array}\right)
$$

To clarify further the exchange of probability flux between $\mathcal{T}$ and $\widetilde{\mathcal{T}}$, note that (1a) and (2a) at $C=b$ combine to

$$
\frac{\partial \mathbf{A}(b, t)}{\partial t}+\frac{\partial \mathbf{D}(t)}{\partial t}=\mathbf{D}(t) \widetilde{Q}+\mathbf{A}(b, t) Q .
$$

We see that the derivatives of $\mathbf{A}$ and $\mathbf{D}$ with respect to time satisfy a conservation principle similar to the forward equations of a continuous-time Markov process. As (3) has a more familiar form than the equivalent equation (2a), we prefer to work with the former rather than the latter. 


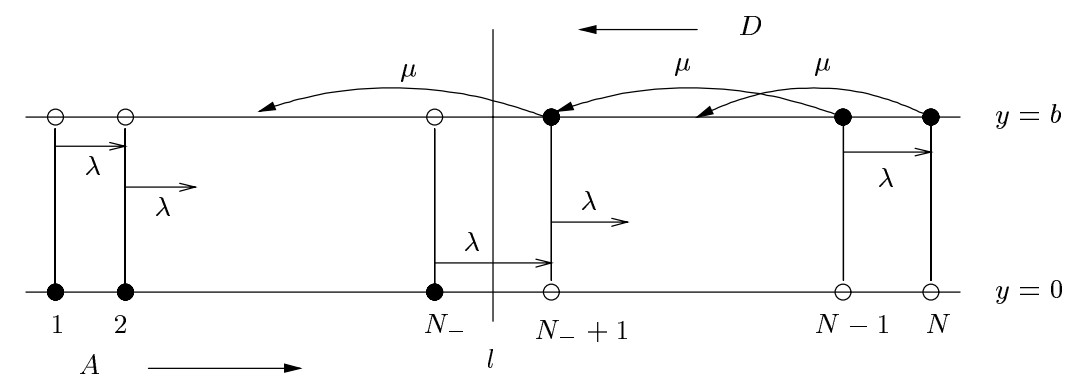

Figure 3: The figure shows the state space and the boundary conditions of $\mathbf{A}(y)$ and $\mathbf{D}$. The open circles at the points $(i, 0)$ for $i \in \mathcal{S}_{+}$indicate that $A_{i}(0)=0$. Likewise we have that $D_{i}=0$ when $i \in \mathcal{S}_{-}$which is shown by the open circles at $(i, b)$. Furthermore $A_{i}(y \geq 0)>0$, when $i \in \mathcal{S}_{-}$, and $D_{i}>0$, when $i \in \mathcal{S}_{+}$ respectively, which is depicted by a filled circle.

\subsection{The Steady State Problem and Boundary Conditions}

We are interested in the steady state behavior of $\{X(t), C(t)\}$. Hence we set $\partial_{t} \mathbf{A}=\partial_{t} \mathbf{D}=0$ in (1a) and (3) and solve the reduced system. It is easily seen that (1a) becomes a set of ordinary linear differential equations

$$
\frac{d \mathbf{A}(y)}{d y} R=\mathbf{A}(y) Q,
$$

and (3) yields balance equations

$$
\mathbf{D} \widetilde{Q}+\mathbf{A}(b) Q=\mathbf{0} .
$$

$\mathbf{A}(y)$ and $\mathbf{D}$ have to satisfy other boundary conditions besides (4b). In fact, we observe that the buffer cannot be full (empty) when $X \in \mathcal{S}_{-}\left(X \in \mathcal{S}_{+}\right)$. Hence,

$$
\mathbf{D}_{-}=\mathbf{0} \quad \text { and } \quad \mathbf{A}_{+}(0)=\mathbf{0} .
$$

Figure 3 shows an overview of the state space $\mathcal{S} \times[0, b]$ with the boundary conditions and the source transitions.

The number of conditions can be seen to be $2 N$ : (5) yields exactly $N$ conditions; it is shown in A.1 that the balance equations (4b) provide $N-1$ conditions; finally, the requirement that $\sum_{i}\left(A_{i}(b)+D_{i}\right)=1$ fixes a scaling. On the other hand, in Section 3.4.1 we show that the number of unknown parameters involved in the solution of (4a) is $N$. The vector $\mathbf{D}$ consists of just $N$ constants. Clearly, the number of unknowns and the number of conditions match, so that we may conclude that the system is well defined.

\subsection{Solving the Stationary System}

Now we solve the system of differential equations (4a) together with the balance equations (4b) such that the boundary conditions (5) are satisfied. First, in Section 3.4.1, we reduce (4a) to an $N$-dimensional eigenvalue problem and establish explicit expressions for the eigenvalues and corresponding left eigenvectors. The second step, Section 3.4.2, relates the eigensystem to the balance equations, includes the boundary conditions, and, finally, provides the solution to the entire system.

\subsubsection{The Eigenvalues and Eigenvectors}

The system of ordinary differential equations (4a) can be written as

$$
\frac{\partial \mathbf{A}(y)}{\partial y}=\mathbf{A}(y) Q R^{-1} .
$$


The inverse of $R$ exists as, by assumption, $l \neq i r$ for any $i \in \mathcal{S}$. The eigenvalues $\theta_{i}$ follow immediately from the upper-triangularity of $Q R^{-1}$ : they simply form the diagonal, so that

$$
\theta_{i}=\frac{\lambda}{l-i r}, \quad 1 \leq i<N,
$$

and $\theta_{N}=0$. Obviously, $N_{-}$eigenvalues are negative, and $N_{+}-1$ are positive.

The eigenvalues are distinct so that the general solution of is of the form

$$
\mathbf{A}(y)=\sum_{i=1}^{N} a_{i} \phi_{i} e^{\theta_{i} y}
$$

where the row vectors $\phi_{i}$ are the left eigenvectors of the matrix $Q R^{-1}$ associated with the eigenvalue $\theta_{i}$, i.e.,

$$
\theta_{i} \phi_{i}=\phi_{i} Q R^{-1} \text {. }
$$

$\mathbf{A}(y)$ can be written succinctly in matrix form. To this end, let

$$
\boldsymbol{\theta}=\left(\theta_{1}, \ldots, \theta_{N}\right)
$$

be the vector of eigenvalues,

$$
\Theta(y)=\exp (\operatorname{diag}(\boldsymbol{\theta}) y),
$$

and $\Phi$ the matrix of eigenvectors, i.e.,

$$
\Phi=\left(\begin{array}{c}
\phi_{1} \\
\vdots \\
\phi_{N}
\end{array}\right) .
$$

Now $\mathbf{A}(y)$ becomes

$$
\mathbf{A}(y)=\mathbf{a} \Theta(y) \Phi,
$$

where the coefficients vector a contains the $N$ unknowns which were to be found in Section 3.3.

To determine the eigenvectors, we notice that, again because of the upper-triangularity of $Q R^{-1}$, the $j$-th component of the eigenvector $\phi_{i}$ should be zero when $j<i$, i.e, $\phi_{i j}=0$ if $j<i$. The other components of $\phi_{i}$ can be found from a recursion which follows from the fact that the only non-zero entries of $Q R^{-1}$, besides the diagonal, appear one above the diagonal. Hence, for $\phi_{i}$ and $i<j<N$,

$$
\phi_{i j}=\frac{1}{j-i} \frac{l-i r}{r} \phi_{i, j-1}=\frac{1}{(j-i) !}\left(\frac{l-i r}{r}\right)^{j-i} \phi_{i i},
$$

and when $i<j=N$,

$$
\phi_{i N}=\frac{1}{(N-i-1) !}\left(\frac{l-i r}{r}\right)^{N-i} \frac{r}{N r-l} \phi_{i i} .
$$

The numbers $\phi_{i i}$ can be chosen arbitrarily as the coefficients vector a ensures that the boundary conditions be met. We therefore set $\phi_{i i}=1,1 \leq i<N$. Furthermore, $\theta_{N}=0$ so that defining $\phi_{N N}=1$ is allowed. As a result, the matrix $\Phi$ is upper-triangular with 1's on the diagonal. Note that the eigenvectors are all linearly independent and span the eigenspace of our boundary value problem for all $y \in(0, b)$.

\subsubsection{Finding $\mathbf{A}(y)$ and $\mathrm{D}$}

It remains to express $\mathbf{a}$ and $\mathbf{D}$ in terms of the boundary conditions (5) and the balance equations (4b). We start with partitioning $\Phi$ according to

$$
\Phi=\left(\begin{array}{c|c}
\Phi^{--} & \Phi^{-+} \\
\hline \Phi^{+-} & \Phi^{++}
\end{array}\right) .
$$


Here $\Phi^{--}$is the $N_{-} \times N_{-}$upper left corner of $\Phi, \Phi^{+-}$is the $N_{+} \times N_{-}$lower left corner, etc. Clearly, $\Phi^{+-} \equiv 0$, since $\Phi$ is upper triangular. Furthermore $\Phi^{--}$and $\Phi^{++}$are invertible, as is apparent from the fact that $\Phi_{i i}=1$ for $1 \leq i \leq N$ and, again, by upper triangularity. We partition the matrix $\Theta(y)$ as well:

$$
\Theta(y)=\left(\begin{array}{cc}
\Theta^{--}(y) & 0 \\
0 & \Theta^{++}(y)
\end{array}\right)
$$

With these partitions we can use the condition $\mathbf{A}_{+}(0)=\mathbf{0}$ to express $\mathbf{a}_{+}$in terms of $\mathbf{a}_{-}$. From (7), and noting that $\Theta(0)$ is the identity,

$$
\begin{aligned}
\mathbf{0} & =[\mathbf{a} \Theta(0) \Phi]_{+} \\
& =\mathbf{a}_{-} \Phi^{-+}+\mathbf{a}_{+} \Phi^{++},
\end{aligned}
$$

from which

$$
\mathbf{a}_{+}=-\mathbf{a}_{-} \Phi^{-+}\left(\Phi^{++}\right)^{-1} \text {. }
$$

Now we can write $\mathbf{A}(b)$ in terms of $\mathbf{a}_{-}$. To this end, introduce the $N_{-} \times N_{+}$matrix

$$
\Psi=\left(\Theta^{--}(b)\right)^{-1} \Phi^{-+}\left(\Phi^{++}\right)^{-1} \Theta^{++}(b),
$$

so that

$$
\begin{aligned}
\mathbf{A}(b) & =\left(\mathbf{a}_{-}, \mathbf{a}_{+}\right) \Theta(b) \Phi \\
& =\mathbf{a}_{-} \Theta^{--}(b)\left(\Phi^{--}-\Psi \Phi^{+-}, \Phi^{-+}-\Psi \Phi^{++}\right) \\
& =\mathbf{a}_{-} \Theta^{--}(b)\left(\Phi^{--}, \Phi^{-+}-\Psi \Phi^{++}\right)
\end{aligned}
$$

where the last step follows from the fact that $\Phi^{+-} \equiv 0$. The advantage of introducing the matrix $\Psi$ is that both $\left(\Theta^{--}\right)^{-1}(b)$ and $\Theta^{++}(b)$ contain (very) small entries. Moreover, while $\mathbf{a}_{-}$can have very small entries, e.g., $10^{-20}$, the entries of the vector $\mathbf{a}_{-} \Theta^{--}(b)$ are in comparison roughly of order 1 . The numerical analysis becomes much stabler when we compute the latter vector instead of $\mathbf{a}_{-}$itself.

With the boundary condition $\mathbf{D}_{-}=\mathbf{0}$ and the above expression for $\mathbf{A}(b)$ we solve for $\mathbf{a}_{-} \Theta^{--}(b)$ and $\mathbf{D}_{+}$ by means of the balance equation $\mathbf{A}(b) Q+\left(\mathbf{0}, \mathbf{D}_{+}\right) \widetilde{Q}=\mathbf{0}$. This final equation is of the form

$$
\left(\mathbf{a}_{-} \Theta^{--}(b), \mathbf{D}_{+}\right) M=\mathbf{0}
$$

for some matrix $M$. The proper expressions for $\mathbf{A}(b)$ and $\mathbf{D}$ can now be found with (10) and the scaling

$$
\sum_{i}\left(A_{i}(b)+D_{i}\right)=1
$$

\subsection{Results for the Case $N=2$}

We present analytical results for a single source with two states. Although a two-state feedback system is not quite a realistic model for TCP, it allows us, due to its relative simplicity, to make a few observations with respect to limiting behavior of flow control. For systems with more states the analytical expressions soon become unwieldy. Therefore we analyze larger models numerically. The results of the numerical study are presented in the accompanying paper [5].

Assuming $r<l<2 r$ straightforward calculations yield the probability of finding the system in state 1 :

$$
A_{1}(b)=\frac{\mu}{\lambda+\mu}\left[1+\frac{\mu}{\lambda+\mu} \frac{l-r}{2 r-l}\left(1-e^{-b \theta_{1}}\right)\right]^{-1},
$$

with $\theta_{1}=\lambda /(l-r)$. The fraction of time the buffer is congested is

$$
\begin{aligned}
D_{2} & =\frac{\lambda}{\mu} A_{1}(b) \\
& =\frac{\lambda}{\lambda+\mu}\left[1+\frac{\mu}{\lambda+\mu} \frac{l-r}{2 r-l}\left(1-e^{-b \theta_{1}}\right)\right]^{-1},
\end{aligned}
$$


and by the boundary conditions: $D_{1}=0$. For $\mathbf{A}(y)$ we obtain:

$$
\begin{aligned}
\mathbf{A}(y) & =\left(a_{1}, a_{2}\right) \Theta(y) \Phi \\
& =A_{1}(b)\left(e^{\theta_{1}(y-b)}, \frac{l-r}{2 r-l}\left[e^{\theta_{1}(y-b)}-e^{-\theta_{1} b}\right]\right) .
\end{aligned}
$$

An interesting limiting case is $b=0$. Then $A_{1}(b)=\mu /(\lambda+\mu)$ and $D_{2}=\lambda /(\lambda+\mu)$. Clearly, this is the stationary distribution of a two-state Markov chain with exponential holding times with parameters $\lambda$ and $\mu$, respectively. This behavior is to be expected: from the boundary conditions (5) we have that $A_{2}(0)=0$ implying that as soon as the source makes a transition from state 1 to 2 , the buffer will be full (recall that $b=0)$.

The same limit results when $l \downarrow r$, in which case the buffer never empties. On the other hand, when $l \uparrow 2 r$, the buffer never becomes full, so that $A_{1}(b) \downarrow 0$, and thereby $D_{2} \downarrow 0$ as well.

It is seen that $A_{1}(b)$ and $D_{2}$ decrease monotonically as functions of $b$, and in the limit

$$
\lim _{b \rightarrow \infty} A_{1}(b)=\frac{\mu}{\lambda+\mu} \cdot\left[1+\frac{\mu}{\lambda+\mu} \cdot \frac{l-r}{2 r-l}\right]^{-1} .
$$

The throughput, which we define as

$$
\gamma=r \sum_{i \in \mathcal{S}} i A_{i}(b)
$$

can be expressed in terms of $A_{1}(b)$ :

$$
\gamma=r A_{1}(b)+2 r A_{2}(b)=r\left[A_{1}(b)+2\left(1-A_{1}(b)-D_{2}\right)\right]=r\left(2-A_{1}(b) \frac{2 \lambda+\mu}{\mu}\right) .
$$

Note that we assume that only the data that is sent while the buffer is not congested contributes to the throughput. For a motivation for this choice we refer to [5].

Some remarks concerning the numerical analysis are in place. Replacing the analytic expressions of the entries of $M$, implicitly defined in (11), by their numerical values, introduces rounding errors. As a result, $M$ is no longer singular, so that it is no longer straightforward to find its left null vector. However, the singular values of $M$ computed by the Singular Value Decomposition algorithm, see e.g. [6, 7], were typically in the order of one, except the last, smallest, value, which was near to 0 up to machine precision. The corresponding left singular vector is then the natural candidate solution, up to a scaling factor. The computation of the inverse of $\Theta^{--}(b)$ caused some problems as well. The entries $\Theta_{i}^{--}(b)$ can become very large when $i$ such that $|i r-l|$ small , thereby making the numerical inversion unstable. The solution is simply to compute $\Theta(-b)$ in $(6)$ and take the upper left block of this matrix.

\section{A Two-Source Model}

Before deriving the Kolmogorov differential equations in Section 4.2, we describe the state space of the entire system in more detail in Section 4.1. Section 4.3 discusses the boundary conditions. In the subsequent two sections, Sections 4.4 and 4.5 we present the solution of the two-point boundary value problem. In Section 4.6 we mention some of the results presented in [5]. Finally, in Section 4.7, we extend the analysis of the two-source case to the multiple-source case.

\subsection{States}

In Section 2 we introduced the state space for a multi-source model; here we specialize to two sources.

Let us consider the evolution in time of the processes $\{\mathbf{X}(t)\}=\left\{X_{1}(t), X_{2}(t)\right\}$ and $\{C(t)\}$. We observe that in the course of time, the system is in one of four mutually exclusive 'modes', which do not necessarily occur consecutively in time: 
1. In a congested state, i.e., $C(t)=b$, both sources wait for negative feedback signals before they decrease their rate.

2. After congestion occurred, a downward transition of source 1 removes the congestion from the buffer. Now source 1 can start increasing its rate, while source 2 still has to make a transition downward.

3. After congestion, a transition of source 2 removes the congestion from the buffer.

4. Neither of the two sources is in a congested state, so both sources can increase their rate.

Clearly, the state space of the entire system is not completely specified by just $\mathbf{X}(t)$ and $C(t)$. To distinguish between these four cases we introduce an indicator function $\mathbf{I}(t)=\left(I_{1}(t), I_{2}(t)\right)$. When $I_{i}(t)=1$, source $i$ is in congestion, that is, it should make a transition downwards as its rate was too high at some point in time. When $I_{i}(t)=0$, all data of source $i$ arrives correctly at the destination and is acknowledged. The states $(1,1),(0,1),(1,0)$ and $(0,0)$ correspond to the above four cases, respectively. For notational brevity we address the states of $\mathbf{I}(t)$ slightly differently, and define

$$
I(t)= \begin{cases}0 & \text { if } \mathbf{I}(t)=(0,0), \text { i.e., neither source is in congestion, } \\ 1 & \text { if } \mathbf{I}(t)=(1,0), \text { i.e., just source } 1 \text { is in congestion, } \\ 2 & \text { if } \mathbf{I}(t)=(0,1), \text { i.e., just source } 2 \text { is in congestion, } \\ 3 & \text { if } \mathbf{I}(t)=(1,1), \text { i.e., both sources are in congestion. }\end{cases}
$$

We split the state space $\mathcal{S} \times[0, b) \times \mathcal{I}$ accordingly in four subsets

$$
\begin{aligned}
\mathcal{T}_{0} & =\mathcal{S} \times[0, b) \times 0 \\
\mathcal{T}_{1} & =\mathcal{S} \times[0, b) \times 1 \\
\mathcal{T}_{2} & =\mathcal{S} \times[0, b) \times 2 \\
\widetilde{\mathcal{T}} & =\mathcal{S} \times\{b\} \times 3 .
\end{aligned}
$$

Figure 4 shows how these subsets of $\mathcal{S} \times[0, b] \times \mathcal{I}$ communicate.

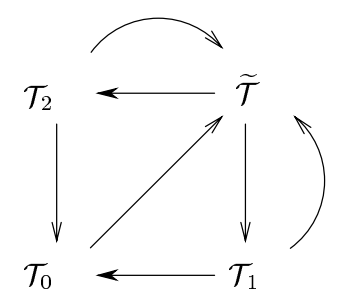

Figure 4: The transitions between the four subspaces are indicated by the arrows. Note that there is no direct transition from $\widetilde{\mathcal{T}}$ to $\mathcal{T}_{0}$. This is a consequence of the boundary conditions, to be discussed in Section 4.3.

With these subsets we associate, in turn, functions. On $\tilde{\mathcal{T}}$ we have

$$
D_{i j}(t)=\mathbb{P}\{\mathbf{X}(t)=(i, j), C(t)=b, I(t)=3\},
$$

and on $\mathcal{T}_{k}$, for $k \in\{0,1,2\}$ and $y<b$,

$$
A_{i j k}(y, t)=\mathbb{P}\{\mathbf{X}(t)=(i, j), C(t) \leq y, I(t)=k\} .
$$

As in the single-source case we distinguish between under- and overload states, i.e.,

$$
\begin{aligned}
& \mathcal{S}_{-}=\left\{(i, j) \in \mathcal{S} \mid r_{i j}<0\right\} \\
& \mathcal{S}_{+}=\left\{(i, j) \in \mathcal{S} \mid r_{i j}>0\right\},
\end{aligned}
$$

where $r_{i j}=i r_{1}+j r_{2}-l$. Here we assume as well that $\mathcal{S}=\mathcal{S}_{1} \cup \mathcal{S}_{+}$, i.e., there are no $(i, j) \in \mathcal{S}$ such that $r_{i j}=0$. As a reminder, $N=|\mathcal{S}|=\left|\mathcal{S}_{1}\right|\left|\mathcal{S}_{2}\right|=N_{1} N_{2}, N_{-}=\left|\mathcal{S}_{-}\right|$, and $N_{+}=N-N_{-}$. Finally, we need the restriction of vectors, $\mathbf{x}$ say, to be indicated by $\mathbf{x}^{-}$to the region $\mathcal{S}_{-}$, etc. 


\subsection{The Differential Equations}

We start by deriving the forward equations that govern the dynamics of the joint process $\{\mathbf{X}(t), C(t), I(t)\}$. Then we cast these in matrix form by means of Kronecker products and sums. In the last part of this section we provide some more explanation about the specific structure of the involved generators.

The derivation of the forward equations is similar to the single source case. The only difference is that we have to incorporate the fact that now two sources can change state, and that the communication between the states is more complicated, as shown by Figure 4. With this in mind, we get, for $0<y<b$ and up to $o(\Delta t)$

$$
\begin{aligned}
A_{i j 0}(y, t+\Delta t)= & \left(1-\left(\lambda_{1}+\lambda_{2}\right) \Delta t\right) A_{i j 0}\left(y-r_{i j} \Delta t, t\right) \\
& +\lambda_{1} \Delta t A_{i-1, j, 0}(y, t)+\lambda_{2} \Delta t A_{i, j-1,0}(y, t) \\
& +\mu_{1} \Delta t\left[A_{2 i, j, 1}(y, t)+A_{2 i+1, j, 1}(y, t)\right] \\
& +\mu_{2} \Delta t\left[A_{i, 2 j, 2}(y, t)+A_{i, 2 j+1,2}(y, t)\right] \\
A_{i j 1}(y, t+\Delta t)= & \left(1-\left(\mu_{1}+\lambda_{2}\right) \Delta t\right) A_{i j 1}\left(y-r_{i j} \Delta t, t\right)+\lambda_{2} \Delta t A_{i, j-1,1}(y, t) \\
A_{i j 2}(y, t+\Delta t)= & \left(1-\left(\mu_{2}+\lambda_{1}\right) \Delta t\right) A_{i j 2}\left(y-r_{i j} \Delta t, t\right)+\lambda_{1} \Delta t A_{i-1, j, 1}(y, t) .
\end{aligned}
$$

(As in the single-source case, we are not too precise about the behavior at the boundaries $i=1, j=1$, $y=0, A_{i j k} \equiv 0,(i, j) \notin \mathcal{S}$. etc.) For $D(t)$ we find for $(i, j) \in \mathcal{S}_{+}$,

$$
\begin{aligned}
D_{i j}(t+\Delta t)= & \left(1-\left(\mu_{1}+\mu_{2}\right) \Delta t\right) D_{i j}(t) \\
& +\mu_{1} \Delta t\left(D_{2 i, j}(t)+D_{2 i+1, j}(t)\right)+\mu_{2} \Delta t\left(D_{i, 2 j}(t)+D_{i, 2 j+1}(t)\right) \\
& +\left(1-\left(\lambda_{1}+\lambda_{2}\right) \Delta t\right) \sum_{k=0}^{2}\left(A_{i j k}(b, t)-A_{i j k}\left(b-r_{i j} \Delta t, t\right)\right) .
\end{aligned}
$$

On $\mathcal{S}_{-}$the last sum should be replaced by $\sum_{k=1}^{2}\left(A_{i j k}(b, t)-A_{i j k}\left(b+r_{i j} \Delta t, t\right)\right)$ because on $\mathcal{S}_{-}$there is a net outflux from $\tilde{\mathcal{T}}$ to $\mathcal{T}_{1}$ and $\mathcal{T}_{2}$, but not to $\mathcal{T}_{0}$, as is shown in Figure 4 .

In the limit $\Delta t \rightarrow 0$ we get a system of partial differential equations on $\mathcal{T}_{0}$ :

$$
\begin{aligned}
\frac{\partial A_{i j 0}(y, t)}{\partial t}+r_{i j} \frac{\partial A_{i j 0}(y, t)}{\partial y}= & \lambda_{1}\left[A_{i-1, j, 0}(y, t)-A_{i j 0}(y, t)\right] \\
& +\lambda_{2}\left[A_{i, j-1,0}(y, t)-A_{i j 0}(y, t)\right] \\
& +\mu_{1}\left[A_{2 i, j, 1}(y, t)+A_{2 i+1, j, 1}(y, t)\right] \\
& +\mu_{2}\left[A_{i, 2 j, 2}(y, t)+A_{i, 2 j+1,2}(y, t)\right],
\end{aligned}
$$

on $\mathcal{T}_{1}$ and $\mathcal{T}_{2}$ :

$$
\begin{aligned}
& \frac{\partial A_{i j 1}(y, t)}{\partial t}+r_{i j} \frac{\partial A_{i j 1}(y, t)}{\partial y}=\lambda_{2}\left[A_{i, j-1,1}(y, t)-A_{i j 1}(y, t)\right]-\mu_{1} A_{i j 1}(y, t) \\
& \frac{\partial A_{i j 2}}{\partial t}(y, t)+r_{i j} \frac{\partial A_{i j 2}}{\partial y}(y, t)=\lambda_{1}\left[A_{i-1, j, 2}(y, t)-A_{i j 2}(y, t)\right]-\mu_{2} A_{i j 2}(y, t)
\end{aligned}
$$

and on $\widetilde{\mathcal{T}}$ :

$$
\begin{aligned}
\frac{\partial D_{i j}(t)}{\partial t}= & \mu_{1}\left[D_{2 i, j}(t)+D_{2 i+1, j}(t)-D_{i j}(t)\right] \\
& +\mu_{2}\left[D_{i, 2 j}(t)+D_{i, 2 j+1}(t)-D_{i j}(t)\right]+r_{i j} \sum_{k=0}^{2} \frac{\partial A_{i j k}(b, t)}{\partial y} .
\end{aligned}
$$

(The summation here runs from $k=0$ to 2 for all $(i, j) \in \mathcal{S}$, instead of from $k=1$ to 2 on $(i, j) \in \mathcal{S}_{-}$. This will be clarified once we derive the boundary conditions in (19).)

As in the single-source case our main concern is the steady state performance: we set $\partial_{t} \mathbf{A}_{k}=0$ and $\partial_{t} \mathbf{D}=0$, and drop the dependency on $t$. The resulting equations for the stationary state can be written 
conveniently with Kronecker products and sums ${ }^{1}$, see e.g. [12]. For this purpose we associate with the matrix $A_{0}$, with entries $A_{i j 0}$, a vector valued function $\mathbf{A}_{0}(y)$ by 'stacking' the rows of the matrix $A_{0}$ into one long vector, i.e.,

$$
\mathbf{A}_{0}=\left(A_{110}, \ldots, A_{1 N_{2} 0}, A_{210}, \ldots, A_{N_{1} N_{2} 0}\right),
$$

and construct similar vectors $\mathbf{A}_{1}, \mathbf{A}_{2}, \mathbf{D}$. We also introduce matrices $Q_{i}, \widetilde{Q}_{i}$, and $R_{i}$, respectively, for $i \in$ $\{1,2\}$, in the form of equations (1b), (1c) and (2b), with $\lambda_{i}, \mu_{i}, r_{i}$ replacing $\lambda, \mu$, and $r$. Finally, we define the rate matrix

$$
R=R_{1} \oplus R_{2}-l I_{N_{1}} \otimes I_{N_{2}}
$$

with $I_{N_{i}}$ the $N_{i} \times N_{i}$ identity matrix, and modified generators

$$
\begin{aligned}
& \widetilde{Q}_{i}^{D}=-\mu_{i} I_{N_{i}}, \\
& \widetilde{Q}_{i}^{O}=\widetilde{Q}_{i}-\widetilde{Q}_{i}^{D} .
\end{aligned}
$$

The first matrix $\widetilde{Q}_{i}^{D}$ is equal to the diagonal of $\widetilde{Q}_{i}$ apart from $\left(\widetilde{Q}_{i}^{D}\right)_{11}$, which is equal to $-\mu_{i}$, while $\left(\widetilde{Q}_{i}\right)_{11}=0$. We will explain this point presently. The matrix $\widetilde{Q}_{i}^{O}$ contains the off-diagonal non-zero elements.

Equations (12a)-(12b) can now be cast into matrix form:

$$
\begin{aligned}
\frac{d \mathbf{A}_{0}}{d y}(y) R & =\mathbf{A}_{0}(y)\left(Q_{1} \oplus Q_{2}\right)+\mathbf{A}_{1}(y)\left(\widetilde{Q}_{1}^{O} \otimes I_{N_{2}}\right)+\mathbf{A}_{2}(y)\left(I_{N_{1}} \otimes \widetilde{Q}_{2}^{O}\right) \\
\frac{d \mathbf{A}_{1}}{d y}(y) R & =\mathbf{A}_{1}(y)\left(\widetilde{Q}_{1}^{D} \oplus Q_{2}\right) \\
\frac{d \mathbf{A}_{2}}{d y}(y) R & =\mathbf{A}_{2}(y)\left(Q_{1} \oplus \widetilde{Q}_{2}^{D}\right) .
\end{aligned}
$$

We observe that $\mathbf{A}_{1}$ and $\mathbf{A}_{2}$ satisfy homogeneous differential equations similar to the single-source equation (4a). The differential equations for $\mathbf{A}_{0}$ contain two inhomogeneous terms related to $\mathbf{A}_{1}$ and $\mathbf{A}_{2}$. For (12c) we get,

$$
\mathbf{0}=\mathbf{D}\left(\widetilde{Q}_{1} \oplus \widetilde{Q}_{2}\right)+\sum_{k=0}^{2} \frac{d \mathbf{A}_{k}(b)}{d y} R .
$$

Comparable to (4b) we combine (14) and (15) to a set of balance equation:

$$
\mathbf{A}_{0}(b)\left(Q_{1} \oplus Q_{2}\right)+\mathbf{A}_{1}(b)\left(\widetilde{Q}_{1} \oplus Q_{2}\right)+\mathbf{A}_{2}(b)\left(Q_{1} \oplus \widetilde{Q}_{2}\right)+\mathbf{D}\left(\widetilde{Q}_{1} \oplus \widetilde{Q}_{2}\right)=\mathbf{0} .
$$

The number of unknowns now follows easily: $4 N$ coefficients need to be found. Each $\mathbf{A}_{i}$ depends on $N$ coefficients, and $\mathbf{D}$ carries an additional $N$ unknowns. Hence, to solve this system we have to identify, in total, $4 N-1$ boundary conditions. With these the solution will be uniquely specified by the final scaling requirement

$$
\sum_{i=1}^{N_{1}} \sum_{j=1}^{N_{2}}\left(A_{i j 0}(b)+A_{i j 0}(b)+A_{i j 2}(b)+D_{i j}\right)=1 .
$$

\subsection{Boundary Conditions}

In (14) we presented a system of differential equations that describe (part of) the dynamics of the two-source system. To fix the solutions we need to identify boundary conditions. It turns out that a sufficient number caqn be found to specify the solutions up to a scaling factor.

Part of the boundary conditions are analogous to the single-source boundary conditions (5):

$$
\begin{aligned}
\mathbf{A}_{k}^{+}(0) & =\mathbf{0}, \quad \text { for } k \in\{0,1,2\} \\
\mathbf{D}_{-} & =\mathbf{0} .
\end{aligned}
$$

\footnotetext{
${ }^{1}$ Given two matrices $A \in \mathbb{R}^{m \times m}$ and $B \in \mathbb{R}^{n \times n}$, the Kronecker product is written as $A \otimes B$, and Kronecker sum is $A \oplus B=A \otimes I_{n}+I_{m} \otimes B$, with $I_{n}\left(I_{m}\right)$ the identity matrix on $\mathbb{R}^{n}\left(\mathbb{R}^{m}\right)$.
} 
Apparently there are $3 N_{+}+N_{-}$of these conditions.

The conditions (18) put a restriction on the value of the solution at $y=0$ and $y=b$. We can identify more conditions by considering the flux of the solutions on the boudary, i.e., conditions on the derivatives of $\mathbf{A}_{k}$. It is clear that when $X \in \mathcal{S}_{+}$the outflux of $\mathcal{T}_{i}, i \in\{0,1,2\}$, should be the influx to $\tilde{\mathcal{T}}$. In fact, regarded in this way, (15) restricted to $\mathcal{S}_{+}$becomes a flux condition. On $\mathcal{S}_{-}$we distribute the outflux of $\tilde{\mathcal{T}}$ over $\mathcal{T}_{0}, \mathcal{T}_{1}$ and $\mathcal{T}_{2}$ according to:

$$
\begin{aligned}
& \frac{d A_{0, i}(b)}{d y} R=0, \\
& \frac{d A_{1, i}(b)}{d y} R=-\left(\mathbf{D}\left(I_{N_{1}} \otimes \widetilde{Q}_{2}\right)\right)_{i}, \\
& \frac{d A_{2, i}(b)}{d y} R=-\left(\mathbf{D}\left(\widetilde{Q}_{1} \otimes I_{N_{2}}\right)\right)_{i} .
\end{aligned}
$$

These conditions are natural in view of the current setting, i.e., modeling TCP. To see this, consider the first condition. A transition from $\widetilde{\mathcal{T}}$ to $\mathcal{T}_{0}$ requires that both sources make a downward jump at nearly the same moment. Such events clearly are $o(\Delta t)$, so that in the limit $\Delta t \rightarrow 0$ they can be neglected. Hence the probability influx at $y=b$ to $\mathcal{T}_{0}$ should be 0 . The second and third conditions distribute the outflux of $\tilde{\mathcal{T}}$ on $\mathcal{S}_{-}$over $\mathcal{T}_{1}$ and $\mathcal{T}_{2}$ in proportion to the downward rate of the second and first source, respectively. Clearly, (19) provides $3 N_{-}$conditions.

Regarding (15), or rather (16), we prove in Appendix A.2 that this system of equations is of rank $N-1$. On the region $\mathcal{S}_{-}$precisely $N_{-}$of the involved equations are automatically satisfied once the solution for $\mathbf{A}_{i}$ and $\mathbf{D}$ meet (19), but $N_{+}-1$ equations remain.

In effect we now have identified all required boundary conditions. We summarize our findings in the table below.

\begin{tabular}{l|rr} 
Region & $\mathcal{S}_{-}$ & $\mathcal{S}_{+}$ \\
\hline Value conditions (18) & $N_{-}$ & $3 N_{+}$ \\
Flux conditions (16),(19) & $3 N_{-}$ & $N_{+}-1$
\end{tabular}

These conditions clearly sum up to $4 N-1$, so that we may conclude that the system is fully specified up to a scaling factor.

\subsection{The Solution for A}

Our next task is to obtain a general solution of the equations (14). While $\mathbf{A}_{1}$ and $\mathbf{A}_{2}$ satisfy homogeneous differential equations, the vector function $\mathbf{A}_{0}$ is the solution of an inhomogeneous system of linear differential equations. Before establishing the full solution for $\mathbf{A}_{0}$, we concentrate first on solving (14) for $\mathbf{A}_{1}$ and $\mathbf{A}_{2}$ and the homogeneous equation

$$
\frac{d \mathbf{A}_{0}^{\text {hom }}(y)}{d y}=\mathbf{A}_{0}^{\text {hom }}(y)\left(Q_{1} \oplus Q_{2}\right) R^{-1} .
$$

The procedure to find the solutions for the homogeneous equations is completely analogous to the singlesource case as presented in Section 3.4.1; we will here only summarize the results and fix some notation. For $i \in\{0,1,2\}$ denote by $\Phi_{i}, \theta_{i}$ and $\mathbf{a}_{i}$, respectively, the appropriate matrix of eigenvectors, eigenvalues and vector of coefficients. Moreover, let $\Theta_{i}(y)=\exp \left(y \operatorname{diag}\left(\boldsymbol{\theta}_{i}\right)\right)$. Now the solution can be written, for $i \in\{0,1,2\}$, as

$$
\mathbf{A}_{i}^{\text {hom }}(y)=\mathbf{a}_{i} \Theta_{i}(y) \Phi_{i},
$$

where $\mathbf{A}_{1}^{\text {hom }}(y) \equiv \mathbf{A}_{1}(y)$ and $\mathbf{A}_{2}^{\text {hom }}(y) \equiv \mathbf{A}_{2}(y)$.

Let us now work out the particular solution for $\mathbf{A}_{0}$. It can be seen from (14) that its differential equations have the general form

$$
\frac{d \mathbf{x}(t)}{d t}=\mathbf{x}(t) M+\mathbf{f}(t)
$$


where $\mathbf{x}=\left(x_{1}, \ldots, x_{n}\right), \mathbf{f}=\left(f_{1}, \ldots, f_{n}\right)$ and $M$ an $n \times n$ matrix. This type of problems has a well known solution, see e.g. [12],

$$
\mathbf{x}(t)=\mathbf{x}_{0} e^{M t}+\int_{0}^{t} \mathbf{f}(s) e^{-M s} d s e^{M t}
$$

Applying this to $\mathbf{A}_{0}$ yields the expression

$$
\begin{aligned}
\mathbf{A}_{0}(y)=\mathbf{A}_{0}^{\mathrm{hom}}(y) & +\int_{0}^{y} \mathbf{A}_{1}(s)\left(\widetilde{Q}_{1}^{O} \otimes I_{N_{2}}\right) R^{-1} e^{-\left(Q_{1} \oplus Q_{2}\right) R^{-1} s} d s e^{\left(Q_{1} \oplus Q_{2}\right) R^{-1} y} . \\
& +\int_{0}^{y} \mathbf{A}_{2}(s)\left(I_{N_{1}} \otimes \widetilde{Q}_{2}^{O}\right) R^{-1} e^{-\left(Q_{1} \oplus Q_{2}\right) R^{-1} s} d s e^{\left(Q_{1} \oplus Q_{2}\right) R^{-1} y} .
\end{aligned}
$$

The integrals, which should be carried out entry-wise, can be considerably simplified. Using (20) and the property that $\Phi_{0} \exp \left(s\left(Q_{1} \oplus Q_{2}\right) R^{-1}\right)=\Theta_{0}(s) \Phi_{0}$, we obtain

$$
\mathbf{A}_{0}(y)=\left(\mathbf{a}_{0}+\mathbf{a}_{1} M_{1}(y)+\mathbf{a}_{2} M_{2}(y)\right) \Theta_{0}(y) \Phi_{0},
$$

where the matrices $M_{1}(y)$ and $M_{2}(y)$ are defined as

$$
\begin{aligned}
& M_{1}(y)=\int_{0}^{y} \Theta_{1}(s) \Phi_{1}\left(\widetilde{Q}_{1}^{O} \otimes I_{N_{2}}\right) R^{-1} \Phi_{0}^{-1} \Theta_{0}(-s) d s, \\
& M_{2}(y)=\int_{0}^{y} \Theta_{2}(s) \Phi_{2}\left(I_{N_{1}} \otimes \widetilde{Q}_{2}^{O}\right) R^{-1} \Phi_{0}^{-1} \Theta_{0}(-s) d s .
\end{aligned}
$$

Note that now, contrary to (21), the integration is straightforward as $\Theta_{i}(s)$ are diagonal matrices. The actual integration, then, yields for the $i j$-th component of $M_{1}$,

$$
M_{i j 1}(y)=\frac{e^{\left(\theta_{i 1}-\theta_{j 0}\right) y}-1}{\theta_{i 1}-\theta_{j 0}} \cdot\left(\Phi_{1}\left(\widetilde{Q}_{1}^{O} \otimes I_{N_{2}}\right) \Phi_{0}^{-1}\right)_{i j},
$$

with $\theta_{i 1}\left(\theta_{j 0}\right)$ the $i$-th $(j$-th $)$ component of $\boldsymbol{\theta}_{\mathbf{1}}\left(\boldsymbol{\theta}_{\mathbf{0}}\right)$. Similar expressions hold for the entries of $M_{2}(y)$. This form of the matrices $M_{1}(y)$ and $M_{2}(y)$ can be easily evaluated numerically.

We now summarize the complete solution of (14) in matrix form,

$$
\left(\mathbf{A}_{0}(y), \mathbf{A}_{0}(y), \mathbf{A}_{0}(y)\right)=\left(\mathbf{a}_{0}, \mathbf{a}_{1}, \mathbf{a}_{2}\right)\left(\begin{array}{ccc}
I_{N} & 0 & 0 \\
M_{1}(y) & I_{N} & 0 \\
M_{2}(y) & 0 & I_{N}
\end{array}\right)\left(\begin{array}{ccc}
\Theta_{0}(y) \Phi_{0} & & 0 \\
& \Theta_{1}(y) \Phi_{1} & \\
0 & & \Theta_{2}(y) \Phi_{2}
\end{array}\right) .
$$

\subsection{Solving for a and D}

The last step is to express all boundary conditions as a number of linear equations from which to we can compute $\mathbf{a}_{0}, \mathbf{a}_{1}, \mathbf{a}_{2}$ and $\mathbf{D}$. Thus, we are to construct an expression comparable to the single-source equation (11). The work to be done at this point resembles Section 3.4.2 in most respects; we only point out the most important steps.

We start with the boundary conditions (18). Consider $\mathbf{A}_{0}^{+}(0)=\mathbf{0}$, i.e.,

$$
\begin{aligned}
\mathbf{0} & =\mathbf{A}_{0}^{+}(0) \\
& =\mathbf{a}_{0}^{-} \Phi_{0}^{-+}+\mathbf{a}_{0}^{+} \Phi_{0}^{++} .
\end{aligned}
$$

From this we find

$$
\mathbf{a}_{0}^{+}=-\mathbf{a}_{0}^{-} \Phi_{0}^{-+}\left(\Phi_{0}^{++}\right)^{-1}
$$

For $\mathbf{a}_{1}$ and $\mathbf{a}_{2}$ similar expressions hold. Clearly, $\mathbf{a}_{i}^{+}$can be expressed in terms of $\mathbf{a}_{i}^{-}$.

At $y=b$, equations (16), (14) and (19) can be written as

$$
\begin{aligned}
& \mathbf{0}=\mathbf{A}_{0}(b)\left(Q_{1} \oplus Q_{2}\right)+\mathbf{A}_{1}(b)\left(\widetilde{Q}_{1} \oplus Q_{2}\right)+\mathbf{A}_{2}(b)\left(Q_{1} \oplus \widetilde{Q}_{2}\right)+\mathbf{D}\left(\widetilde{Q}_{1} \oplus \widetilde{Q}_{2}\right) \\
& \mathbf{0}=\left(\mathbf{A}_{0}(b)\left(Q_{1} \oplus Q_{2}\right)\right)^{-}+\left(\mathbf{A}_{1}(b)\left(\widetilde{Q}_{1}^{O} \otimes I_{N_{2}}\right)\right)^{-}+\left(\mathbf{A}_{2}(b)\left(I_{N_{1}} \otimes \widetilde{Q}_{2}^{O}\right)\right)^{-} \\
& \mathbf{0}=\left(\mathbf{A}_{1}(b)\left(Q_{1}^{D} \oplus Q_{2}\right)\right)^{-}+\left(\mathbf{D}\left(I_{N_{1}} \otimes \widetilde{Q}_{2}\right)\right)^{-} \\
& \mathbf{0}=\left(\mathbf{A}_{2}(b)\left(Q_{1} \oplus \widetilde{Q}_{2}^{D}\right)\right)^{-}+-\left(\mathbf{D}\left(\widetilde{Q}_{1} \otimes I_{N_{2}}\right)\right)^{-}
\end{aligned}
$$


Note that, by the remark below (16), the first equation fixes only $N_{+}-1$ independent coefficients.

The condition for $\mathbf{D}$ can be easily incorporated, only $\mathbf{D}_{+}$should be found, since by (18) we already have that $\mathbf{D}^{-}=\mathbf{0}$.

As in Section 3.4.2, we partition $\Phi_{i}, \Theta_{i}(y)$, build matrices $\Psi_{i}$, etc. Combining all boundary conditions, we obtain after tedious but straightforward calculations, the final equation

$$
\left(\mathbf{a}_{0}^{-} \Theta_{0}^{--}(b), \mathbf{a}_{1}^{-} \Theta_{1}^{--}(b), \mathbf{a}_{2}^{-} \Theta_{2}^{--}(b), \mathbf{D}_{+}\right) K=\mathbf{0},
$$

where $K$ is a $\left(3 N_{-}+N_{+}\right) \times\left(3 N_{-}+N_{+}\right)$matrix, and $\mathbf{0}$ is of appropriate size. This equation has to be solved numerically, for instance by the Singular Value Decomposition algorithm. Here, as in the single-source case, solving for $\mathbf{a}_{i}^{-} \Theta_{i}^{--}(b)$ is numerically more robust than solving for $\mathbf{a}_{i}^{-}$straightaway. The final result requires normalization according to (17).

\subsection{Results}

In the accompanying paper [5] we analyze the effect of the following parameters on the bias of TCP:

- The feedback times: $\lambda_{1}, \mu_{1}$ and $\lambda_{2}, \mu_{2}$;

- The maximum source rates: $N_{1}$ and $N_{2}$;

- The rate increase factor: $r_{1}$ and $r_{2}$.

The analysis is somewhat hampered by the fact that the matrix $K$ implicitly defined in (24) is ill-conditioned. The main reason is that we have to solve a two-point boundary problem, that is, the conditions at both $y=0$ and $y=b$ have to be taken into account. This fact presents itself explicitly in the matrices that contain the eigenvalues, e.g. (23). The eigenvalues have the form

$$
\exp \left(K \frac{b}{i r_{1}+j r_{2}-l}\right)
$$

for some constant $K$. Obviously they can become very large, and very small, when $b$ is in the order of 10, say, and $i r_{1}+j r_{2} \approx l$. Since the entries of the eigenvector matrices alternate in sign (compare (8)), multiplication with the eigenvalues results in adding large positive and negative numbers, which is well known to be numerically unstable. A second problem, of lesser interest, is that the dimension of the matrix $K$ is much larger than the one of the single-source case so that its left null space is harder to compute. The consequence of these problems is that the parameter ranges suitable for numerical analysis are rather small in comparison to the single-source case, so that we could not analyze all situations we planned for. Still we can make a number of interesting observations for small-sized problems.

For completeness' sake we include a simple expression for the throughput. This can be concisely expressed in terms of Kronecker products. For the first source this becomes

$$
\begin{aligned}
\gamma_{1} & =r_{1} \sum_{j=1}^{N_{2}} \sum_{i=1}^{N_{1}} i\left(A_{i j 0}(b)+A_{i j 2}(b)\right), \\
& =r_{1}\left(\mathbf{A}_{0}(b)+\mathbf{A}_{2}\right)(b) \cdot\left(\left(1,2, \ldots, N_{1}\right) \otimes \mathbf{1}_{N_{2}}\right)^{\prime},
\end{aligned}
$$

where $\mathbf{1}_{N_{1}}=(1, \ldots, 1) \in \mathbb{R}^{N_{1}}$, and for the second source,

$$
\gamma_{2}=r_{2}\left(\mathbf{A}_{0}(b)+\mathbf{A}_{1}\right)(b) \cdot\left(\mathbf{1}_{N_{1}} \otimes\left(1,2, \ldots, N_{2}\right)\right)^{\prime} .
$$

Similar expressions can be found for other performance measures.

\subsection{The Multiple-Source Case}

The multiple-source case can be handled similarly as the two-source case. However, the number of differential equations and boundary conditions grows exponentially in the number of sources. To see this, consider $J$ sources. Each source can be in one of two modes: ascending or descending. The number of different modes is therefore $2^{J}$. Hence we need $2^{J}-1$ equations of type (14), and 1 of type (15). Obviously we need the same number of boundary conditions minus 1. Clearly, the 'curse of dimensionality' strikes, and the problems with the ill-conditioning increase. For these reasons we have not studied the multiple-source case. 


\section{Summary}

In this paper we develop and solve a class of feedback fluid models to analyze the Transport Control Protocol, one of the essential protocols of the Internet. During overflow, the buffer sends negative feedback signals to the sources to reduce the sending rate. Otherwise the buffer sends positive signals to indicate that the sources can increase the rate. First we derive the results of [17] by a slightly different method and apply this to the case that one TCP source uses the buffer. The stationary solution is expressed in terms of eigenvalues and eigenvectors of a system of linear differential equations. We are able to compute closed form expressions for these eigenvectors and eigenvalues due to the relatively simple form of the involved generators. Then we extend the single-source analysis such that multiple source can be handled. In the specific case of two sources, we derive a sufficient number of boundary conditions to determine the solution uniquely, up to a scaling factor. In both the single- and two-source case we provide a numerically efficient procedure to compute the coefficients of the solution of the differential equations.

We refer the interested reader to the accompanying paper [5], where we relate the TCP fluid model to the TCP literature, provide justification for interpreting the output stream of a TCP source as fluid, discuss numerical results and give directions for further research.

\section{A Appendix}

\section{A.1 The Number of Conditions Implied by Equation (3)}

Let us prove that the number of conditions implied by the equation

$$
\mathbf{A}(b) Q+\mathbf{D} \widetilde{Q}=\mathbf{0},
$$

is equal to $N-1$. We do this in two steps. In the first place we notice that a solution of the differential equations (4a) that satisfies the condition $\sum_{i}\left(A_{i}(b)+D_{i}\right)=1$ cannot be trivial, i.e.:

$$
\begin{aligned}
\mathbf{A}(b) Q & \neq \mathbf{0} \\
\mathbf{D} \widetilde{Q} & \neq \mathbf{0},
\end{aligned}
$$

otherwise $d A(y) / d y \equiv \mathbf{0}, \forall y \in(0, b)$. Secondly, without changing the rank of the system (25), we can add the first row of $Q$ to the second, the first and the second row to the third, etc., up to the second to last row. We can perform similar operations on $\widetilde{Q}$. We end up with a modified system of linear equalities

$$
\widetilde{\mathbf{A}}(b)\left(\begin{array}{cccc}
-\lambda & \lambda & & 0 \\
\vdots & & \ddots & \\
-\lambda & 0 & & \lambda \\
0 & 0 & \ldots & 0
\end{array}\right)+\widetilde{\mathbf{D}}\left(\begin{array}{cccc}
0 & 0 & \ldots & 0 \\
\mu & -\mu & & 0 \\
\vdots & & \ddots & \\
\mu & 0 & & -\mu
\end{array}\right)=\mathbf{0} .
$$

(Note that $\mathbf{A}(b)$ and $\mathbf{D}$ change to $\widetilde{\mathbf{A}}(b)$ and $\widetilde{\mathbf{D}}$.) Clearly the first columns of the remaining matrices are linear combinations of the other columns, which span a space of dimension $N-1$. From this we see that (25) contains precisely $N-1$ independent equations.

\section{A.2 The Number of Conditions Implied by equation (16)}

The proof that the number of conditions implied by equation (16):

$$
\mathbf{A}_{0}(b)\left(Q_{1} \oplus Q_{2}\right)+\mathbf{A}_{1}(b)\left(\widetilde{Q}_{1} \oplus Q_{2}\right)+\mathbf{A}_{2}(b)\left(Q_{1} \oplus \widetilde{Q}_{2}\right)+\mathbf{D}\left(\widetilde{Q}_{1} \oplus \widetilde{Q}_{2}\right)=\mathbf{0}
$$

is equal to $N-1$ is similar to the proof for the single-source case, presented above. The only missing point is to show that the left null-spaces of the involved Kronecker sums are one dimensional. This follows in a straightforward manner from the property that the eigenvalues of the Kronecker sum $A \oplus B$, of any two matrices $A \in \mathbb{R}^{m \times m}$ with eigenvalues $\lambda_{1}, \ldots, \lambda_{m}$, and $B \in \mathbb{R}^{n \times n}$ with eigenvalues $\mu_{1}, \ldots, \mu_{n}$, are the $m n$ 
numbers $\lambda_{i}+\mu_{j},[12]$. To apply this property, let us point out that since all matrices, such as $Q_{1}, \widetilde{Q}_{1}$, etc., in this equation are generators, their left null-spaces are one dimensional. Furthermore, all eigenvalues not associated with the null-spaces are negative, as is apparent from the fact that the generators are either upper or lower tridiagonal. Hence, the sum of any two eigenvalues is negative, except when both eigenvalues are zero. From this, and the above property of the eigenvalues of Kronecker sums, the Kronecker sum of, e.g., $Q_{1}$ and $Q_{2}$ can have only one zero eigenvalue. The same reasoning applies of course to the other Kronecker sums, so that we see that the null-spaces of all involved Kronecker sums are one dimensional. To finalize the proof, add rows of the matrices such that a system of equalities of the same form as for the single-source case results.

\section{References}

[1] I.J.B.F. Adan, E.A. van Doorn, J.A.C. Resing, and W.R.W. Scheinhardt. Analysis of a single-server queue interacting with a fluid reservoir. Queueing Systems, 29:313-336, 1998.

[2] D. Anick, D. Mitra, and M.M. Sondhi. Stochastic theory of a data-handling system with multiple sources. Bells System Tech. J., 61(8):1871-1894, 1982.

[3] T. Bonald. Comparison of TCP Reno and TCP Vegas via fluid approximation. Technical Report RR-3563, 1998. http://Citeseer.nj.nec.com/bonald98comparison.html.

[4] P. Brown. Resource sharing of TCP connections with different roundtrip times. In Proc. IEEE INFOCOM, April 2000.

[5] N.D. van Foreest, M.R.H. Mandjes, and W.R.W. Scheinhardt. Performance analysis of heterogeneous interacting TCP sources. Memorandum 1607, Faculty of Mathematical Sciences, University of Twente, Enschede, The Netherlands, 2001.

[6] G. H. Golub and C. van Loan. Matrix Computations. The Johns Hopkins University Press, Baltimore, Maryland, 2nd edition, 1989.

[7] R. A. Horn and C. A. Johnson. Matrix Analysis. Cambridge University Press, Cambridge, 1985.

[8] F.P. Kelly. Charging and rate control for elastic traffic. European Transactions on Telecommunications, $8: 33-37,1997$.

[9] F.P. Kelly, A.K. Maulloo, and D.K.H. Tan. Rate control in communication networks: shadow prices, proportional fairness and stability. Journal of the Operational Research Society, 49:237-252, 1998.

[10] L. Kosten. Stochastic theory of a multi-entry buffer, part 1. Delft Progress Report, Series F, 1:10-18, 1974.

[11] V. Kulkarni. Fluid models for single buffer systems. In J.H. Dshalalow, editor, Frontiers in Queueing. Models and Applications in Science and Engineering, pages 321-338, Boca Raton, Florida, 1997. CRC Press.

[12] P. Lancaster and M. Tismenetsky. The Theory of Matrices with Applications. Academic Press, San Diego, 2nd edition, 1985.

[13] M.R.H. Mandjes, D. Mitra, and W.R.W Scheinhardt. A simple model of network access: feedback adaptation of rates and admission control. To appear in Proc. IEEE INFOCOM '02, 2002.

[14] L. Massoulié and J.W. Roberts. Bandwidth sharing: objectives and algorithms. In Proc. of IEEE INFOCOM'99, 1999.

[15] M. Mathis, J. Semske, J. Mahdavi, and T. Ott. The macroscopic behaviour of the TCP congestion avoidance algorithm. ACM Computer Communication Review, 27(3), July 1997. 
[16] W.R.W. Scheinhardt. Markov-Modulated and Feedback Fluid Queues. PhD thesis, Faculty of Mathematical Sciences, University of Twente, Enschede, The Netherlands, 1998. http://www.ub.utwente. $\mathrm{nl} /$ webdocs/tw/1/t0000008.pdf.

[17] W.R.W. Scheinhardt. Analysis of feedback fluid queues. In Proceedings of the 14th ITC specialists seminar on access networks and systems, pages 215-220, Girona, April 2001.

[18] M. Schwartz. Broadband Integrated Networks. Prentice Hall, 1996.

[19] B. Sericola and B. Tuffin. A fluid queue driven by a Markovian queue. Queueing Systems, 31:253-264, 1999.

[20] T. Stern and A. Elwalid. Analysis of separable Markov-modulated models for information handling systems. Adv. Appl. Prob., March 1991. 